\title{
Effect of the Concentration Gradient on Molecular Alignment by Scanning Wave Photopolymerization
}

\author{
Yoshiaki Kobayashi ${ }^{1,2}$, Ryo Taguchi ${ }^{1,2}$, Norihisa Akamatsu ${ }^{1,2}$, and Atsushi Shishido ${ }^{1,2^{*}}$ \\ ${ }^{1}$ Laboratory for Chemistry and Life Science, Institute of Innovative Research, Tokyo Institute of Technology, \\ 4259 Nagatsutacho, Midori-ku, Yokohama 226-8503, Japan \\ ${ }^{2}$ Department of Chemical Science and Engineering, School of Materials and Chemical Technology, \\ Tokyo Institute of Technology, 2-12-1 Ookayama, Meguro-ku, Tokyo 152-8552, Japan \\ *ashishid@res.titech.ac.jp
}

\begin{abstract}
Macroscopic and precise alignment control of functional molecules can provide increased functionality and enhanced device performance. However, even the most powerful current method has some limitations, such as the need for polarized light and photoresponsive dye molecules. We reported an alignment process based on a new concept of scanning wave photopolymerization (SWaP) that achieves an arbitrary alignment pattern by spatiotemporal light-triggered molecular diffusion. Here, we investigate the effect of crosslinker on molecular alignment by SWaP. We found that the controlled concentration gradient of polymer added with the optimal amount of crosslinker improved the degree of alignment of the liquid-crystalline polymers. SWaP could be employed as a simple and versatile fabrication process for preparing highly functional materials and devices that require alignment control.
\end{abstract}

Keywords: Liquid crystal, Molecular alignment, Polymer, Photopolymerization

\section{Introduction}

Controlling the alignment of various functional molecules such as liquid crystals (LCs) is key for generating a new function and enhancing their performances [1-3]. Therefore, molecular alignment methods play an important role in the development and the production of electronic, photonic, mechanical and biomedical organic devices [2-5]. Although mechanical methods are simple and practical processes for one-dimensional alignment over large areas, alignment patterning is difficult. Furthermore, the mechanical methods cause alignment defects due to dust contamination and static electricity [6].

As an alternative method for overcoming these difficulties, photoalignment method using axisselective photoresponsive dye molecules has been developed [7-10]. A representative photoalignment method uses the photoisomerization of azobenzene, which was first reported by Ichimura and Seki et al [11]. Such significant success of a photoreactive alignment layer led to development of photochemical alignment control, referred to as a "command surfaces". The photoalignment method also was applied to photoresponsive polymer systems such as side-chain LC polymers, supramolecules and block copolymers. However, there still remain some challenges, namely the requirement of polarized light and photoresponsive molecules to control the alignment. In addition to such necessity, the alignment processes become complicated in the induction of complex alignment patterns.

Recently, we reported a dye-free photoalignment process based on a new concept of scanning wave photopolymerization (SWaP) [12-18]. In this method, localized photopolymerization generates a concentration gradient of polymer between irradiated and unirradiated regions, resulting in molecular diffusion [19-21]. The diffusion induces the mass flow, and then the flow aligns LCs at the boundary of both regions. Therefore, this method has far fewer limitations on applicable molecular structures and can provide a complex alignment pattern in a single step. For further development of SWaP as a versatile alignment method, to 
investigate the relation between the concentration gradient of polymer and the induction of molecular alignment is important. In this study, we fabricated LC films with different composition ratios of a monomer and a crosslinker, and then examined their molecular alignment behavior. As a result, we revealed that the SWaP fabricated the highly oriented LC film under the optimal condition.

\section{Experimental}

The chemical structures of materials used in this study are shown in Fig. 1. The photopolymerizable anisotropic monomer, 4-(4-propylcyclohexyl)phenyl-4-\{4-[(3-ethyloxetan-3-yl)methoxy] butoxy benzoate (OXT-M) was employed as previously reported [13]. The isotropic crosslinker, 3-ethyl-3 \{[(3-ethyloxetan-3-yl)methoxy]methyl\} oxetane (OXT-221) was provided by TOAGOSEI Co., Ltd., Tokyo, Japan. The cationic photoinitiator, CPI-210S was provided by San-Apro Ltd., Tokyo, Japan. The surfactant, MEGAFAC R-40 were provided by DIC Corporation Tokyo, Japan. Propyleneglycol monomethyl ether acetate (PGMEA) was purchased from Tokyo Chemical Industry Co., Ltd., Tokyo, Japan.

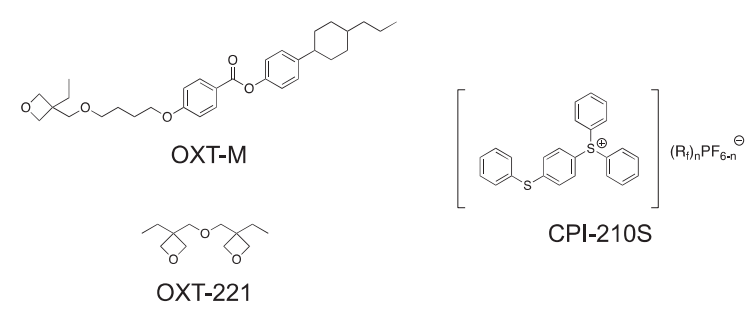

Fig. 1. Chemical structures used in this study.

The mixtures with 99:1, 97:3, and 95:5 molar ratio of OXT-M:OXT-221 (OXT $99: 1, \mathrm{OXT}_{97: 3}$, $\mathrm{OXT}_{95: 5)}$, were prepared by adding CPI-210S to the mixtures at $5 \mathrm{wt} \%$. We then prepared the solutions of $40 \mathrm{wt} \%$ by adding PGMEA, and doped R-40 at $1000 \mathrm{ppm}$. The solutions were stirred at $70{ }^{\circ} \mathrm{C}$ for $10 \mathrm{~min}$, and were spin-coated (MS-A100, MIKASA, Co., Ltd., Tokyo, Japan; spin rate, $800 \mathrm{rpm}$ ) for $30 \mathrm{~s}$ on an ozone-treated glass substrate. The solvent in the coated mixtures was removed at $120{ }^{\circ} \mathrm{C}$ for $2 \mathrm{~min}$. Before photopolymerization, the substrate was cooled to $100{ }^{\circ} \mathrm{C}$ for each mixture, which was their optimized photopolymerization temperature. To induce 1D molecular alignment by photopolymerization, the mixture on the substrate was subsequently irradiated with ultraviolet (UV) light at wavelength of $365 \mathrm{~nm}$ through a $500-\mu \mathrm{m}$ slitted mask from a high-pressure mercury lamp (OPM2-502HQ, USHIO INC., Tokyo, Japan) with glass filters (IRA-25S, UV-D33S, AGC Techno Glass, Co., Ltd., Shizuoka, Japan). The light intensity and the scanning rate were optimized to be $3.2 \mathrm{~mW} / \mathrm{cm}^{2}$ and $20 \mu \mathrm{m} / \mathrm{s}$, respectively (Fig. 2). Finally, the samples were irradiated with UV light (wavelength: $365 \mathrm{~nm}$, light intensity: $10 \mathrm{~mW} / \mathrm{cm}^{2}$ ) for $10 \mathrm{~min}$ to complete photopolymerization without slit to fix the molecular alignment.

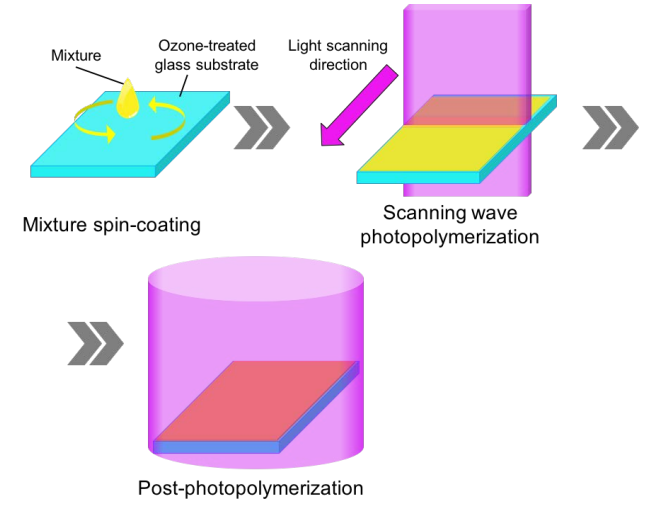

Fig. 2. Schematic illustration of photopolymerization process.

The molecular alignment of the obtained LC polymer films was evaluated with a polarized optical microscope (POM, BX50, Olympus, Tokyo, Japan). The molecular alignment direction was confirmed by polarized UV-visible (vis) absorption spectroscopy. The order parameter $(S)$, which quantitatively showed the degree of the in-plane alignment of mesogens, was calculated from the absorption bands corresponding to phenyl benzoate moieties (302-307 $\mathrm{nm}$ ) by using the following equation:

$$
S=\frac{A_{\|}-A_{\perp}}{A_{\|}+2 A_{\perp}}
$$

where $A_{\|}$and $A_{\perp}$ are defined as the absorbance in which the direction of polarized incident light is parallel and perpendicular to the light scanning direction, respectively.

\section{Results and discussion}

The addition of crosslinker changed the degree of a unidirectional molecular alignment in LC films fabricated by SWaP. We obtained the $\mathrm{OXT}_{99: 1}$, $\mathrm{OXT}_{97: 3}$ and $\mathrm{OXT}_{95: 5}$ films with 1, 3 and $5 \mathrm{~mol} \%$ of crosslinker, respectively, and resultant films showed optical transparency (Figs. 3a, c, e). The POM observation of $\mathrm{OXT}_{99: 1}$ and $\mathrm{OXT}_{97: 3}$ films under crossed polarizers showed a clear contrast by every $45^{\circ}$ rotation (Figs. 3b, d). Obtained images became completely dark when the polarization direction and the light scanning direction were parallel or 
(a)

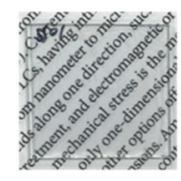

(c)

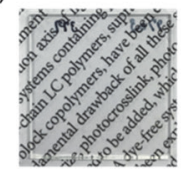

(e)

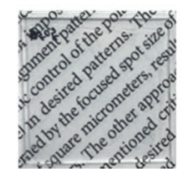

(b)

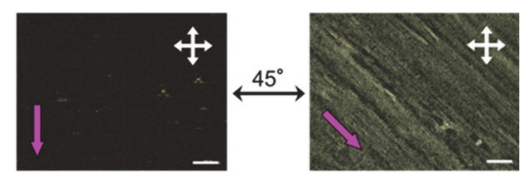

(d)

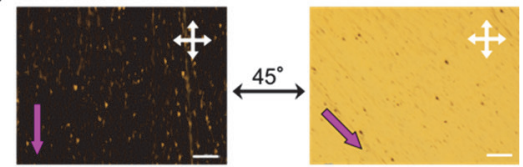

(f)

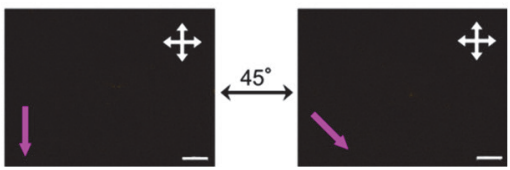

Fig. 3. Photograph and polarized optical micrographs of $\mathrm{OXT}_{99: 1}(\mathrm{a}, \mathrm{b}), \mathrm{OXT}_{97: 3}(\mathrm{c}, \mathrm{d})$ and $\mathrm{OXT}_{95: 5}(\mathrm{e}, \mathrm{f})$. White arrows depict the direction of polarizers. Scale bars, $200 \mu \mathrm{m}$.

perpendicular. We measured birefringences of $\mathrm{OXT}_{99: 1}$ and $\mathrm{OXT}_{97: 3}$ films and then found to be 0.01 and 0.05 , which were determined by POM observation with a Berek compensator. On the other hand, under crossed polarizers, POM image of $\mathrm{OXT}_{95: 5}$ film always showed darkfield even though the stage of POM was rotated by $360^{\circ}$ (Fig. 3f). To further investigate the molecular alignment behavior, we measured the polarized UV-vis absorption spectra and evaluated polar plots of these films. In $\mathrm{OXT}_{99: 1}$ and $\mathrm{OXT}_{97: 3}$ films, the absorption spectra showed a clear difference; the absorbance parallel to the light scanning direction was larger compared to the perpendicular direction (Figs. 4ad). Furthermore, the $S$ values were found to be 0.19 and 0.26 , respectively. This result indicated that the phenyl benzoate moieties in the $\mathrm{OXT}_{99: 1}$ and $\mathrm{OXT}_{97: 3}$ films were in-plane aligned along the light scanning direction. On the contrary, we observed the coincident spectra parallel and perpendicular to the light scanning direction in the $\mathrm{OXT}_{95: 5}$ film, indicating that mesogens in the $\mathrm{OXT}_{95: 5}$ was not aligned (Figs. 4e, f). These results revealed that addition of the appropriate amount of crosslinker is necessary to induce unidirectional molecular alignment by SWaP.

The alignment behavior in $\mathrm{OXT}_{99: 1}, \mathrm{OXT}_{97: 3}$ and $\mathrm{OXT}_{95: 5}$ films is rationalized by the molecular alignment mechanism of SWaP. In SWaP, the spatiotemporal UV light irradiation generates local photopolymerization, which causes the spatial polymer concentration gradient between irradiated

(a)

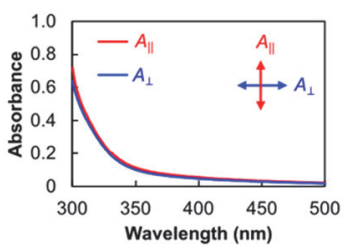

(c)

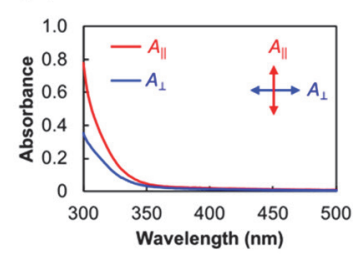

(e)

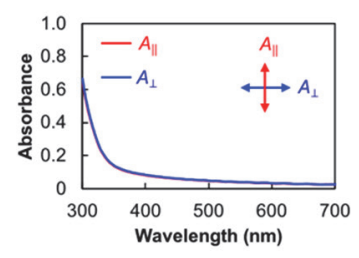

(b)

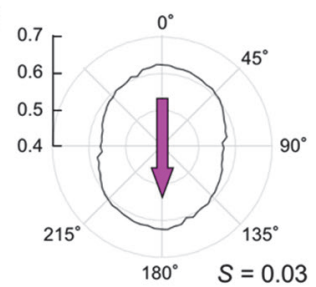

(d)

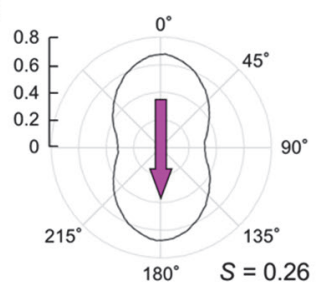

(f)

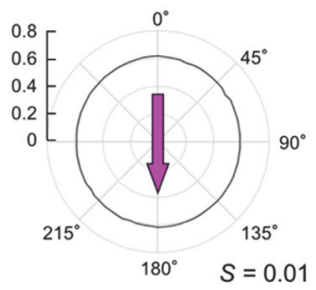

Fig. 4. Polarized UV-vis spectra and polar plots of $\mathrm{OXT}_{99: 1}(\mathrm{a}, \mathrm{b}), \mathrm{OXT}_{97: 3}(\mathrm{c}, \mathrm{d})$ and $\mathrm{OXT}_{95: 5}$ films (e, f). Red and blue arrows show the absorption spectra parallel and perpendicular to the light scanning direction, respectively. Purple arrows show the light scanning direction.

and unirradiated regions. The gradient induces the molecular diffusion. Such diffusion propagates along the direction of scanning light, triggering mass flow. The flow could impose shear stress on polymers, and subsequently align their main chains along the flow direction. Therefore, the concentration gradient is key to the induction of molecular alignment. The number of polymerizable groups in monomer or crosslinker in the systems determines the polymerization rate [20]. Therefore, the increase of the number of polymerizable groups enhances the polymerization rate, that is, the spatial polymer concentration gradient is steep. The $\mathrm{OXT}_{95: 5}$ mixture with a large amount of crosslinker has faster polymerization, and thus finish the polymerization before the molecular alignment is induced. As a result, the alignment in $\mathrm{OXT}_{95: 5}$ film became random. On the other hand, the $\mathrm{OXT}_{99: 1}$ mixture with a small amount of crosslinker decreases the reactivity, and generates the spatial polymer concentration gradient with a gentle slope, leading to the small degree of alignment in $\mathrm{OXT}_{99: 1}$ film. As a result, the alignment behavior depends on the amount of crosslinker due to the created polymer concentration gradient. 


\section{Conclusion}

In summary, we fabricated LC films with various composition ratios of anisotropic monomer and isotropic crosslinker by SWaP, and investigated the effect of the polymer concentration gradient on molecular alignment. SWaP achieved the unidirectional molecular alignment in the LC films with 1 and $3 \mathrm{~mol} \%$ of crosslinker, and the film containing $3 \mathrm{~mol} \%$ of crosslinker showed the highest degree of alignment. On the other hand, the LC film with $5 \mathrm{~mol} \%$ of crosslinker was not aligned. SWaP improves the degree of alignment of LC polymers with the controlled polymer concentration gradient, which is determined by the reactivity of polymerization. We believe that SWaP provides a powerful new method for fabricating highly oriented functional materials and devices.

\section{Acknowledgements}

This work was supported by JSPS KAKENHI Grant Numbers JP18H05422 and JP18K14297. This work was supported by JST CREST Grant Number JPMJCR18I4, Japan. This work was performed under the Cooperative Research Program of "Network Joint Research Center for Materials and Devices".

\section{References}

1. R. Lakes, Nature, 361 (1993) 511.

2. T. J. White and D. J. Broer, Nat. Mater., 14 (2015) 1087.

3. M. O'Neill and S. M. Kelly, Adv. Mater, 15 (2003) 1135.

4. T. Kato, J. Uchida, T. Ichikawa, and T. Sakamoto, Angew. Chem. Int. Ed., 57 (2018) 4355.

5. S. J. Woltman, G. D. Jay and G. P. Crawford, Nat. Mater., 6 (2007) 929.

6. J. M. Geary, J. W. Goodby, A. R. Kmetz, and J. S.
Patel, J. Appl. Phys., 62 (1987) 4100.

7. O. Yaroshchuk and Y. Reznikov, J. Mater. Chem., 22 (2012) 286.

8. A. Shishido, Polym. J., 42 (2010) 525.

9. A. Priimagi, C. J. Barrett, and A. Shishido, J. Mater. Chem. C, 2 (2014) 7155.

10. T. Seki, Bull. Chem. Soc. Jpn., 91 (2018) 1026.

11. K. Ichimura, Y. Suzuki, T. Seki, A. Hosoki, and K. Aoki, Langmuir, 4 (1988) 1214.

12. K. Hisano, Y. Kurata, M. Aizawa, M. Ishizu, T. Sasaki, and A. Shishido, Appl. Phys. Express, 9 (2016) 072601.

13. K. Hisano, M. Aizawa, M. Ishizu, Y. Kurata, W. Nakano, N. Akamatsu, C. J. Barrett, and A. Shishido, Sci. Adv., 3 (2017) e1701610.

14. M. Aizawa, K. Hisano, M. Ishizu, N. Akamatsu, C. J. Barrett, and A. Shishido, Polym. J., 50 (2018) 753.

15. M. Ishizu, M. Aizawa, N. Akamatsu, K. Hisano, S. Fujikawa, C. J. Barrett, and A. Shishido, Appl. Phys. Express, 12 (2019) 041004.

16. M. Aizawa, M. Ota, K. Hisano, N. Akamatsu, T. Sasaki, C. J. Barrett, and A. Shishido, J. Opt. Soc. Am. B, 36 (2019) D47.

17. K. Hisano, M. Ota, M. Aizawa, N. Akamatsu, C. J. Barrett, and A. Shishido, J. Opt. Soc. Am. $B, 36$ (2019) D112.

18. S. Hashimoto, M. Aizawa, N. Akamatsu, T. Sasaki, and A. Shishido, Liq. Cryst., 46 (2019) 1995.

19. V. V. Krongauz and C. C. Legere-Krongauz, Polymer, 34 (1993) 3614.

20. C. F. Van Nostrum, R. J. Nolte, D. J. Broer, T. Fuhrman, and J. H. Wendorff, Chem. Mater, 10 (1998) 135.

21. C. M. Leewis, A. M. de Jong, L. J. van IJzendoorn, and D. J. Broer, J. Appl. Phys., 95 (2004) 4125. 